\title{
Myorelaxation in Special Physical Capability of Athletes
}

\author{
Denisenko Yu. P. \\ Naberezhnye Chelny State Pedagogical University \\ Naberezhnye Chelny, Russia \\ yprof@yandex.ru \\ Akhmetov A.M. \\ Naberezhnye Chelny State Pedagogical University \\ Naberezhnye Chelny, Russia \\ silva76@list.ru
}

\author{
Paramonova D.B. \\ Naberezhnye Chelny State Pedagogical University \\ Naberezhnye Chelny, Russia \\ paramonova.diana2016@yandex.ru \\ Selivyorstova N.N. \\ Naberezhnye Chelny State Pedagogical University \\ Naberezhnye Chelny, Russia \\ silva76@list.ru
}

\begin{abstract}
In our longitudinal studies we have ascertained that unilateral changes of neuromuscular system functional state, particularly, changing velocity of muscles voluntary relaxation under the influence of different adaptogenic factors, depend on the fact that each of such impacts is more or less accompanied by tissue hypoxia and hypoxemia. It causes activation (inclusion) of the whole complex of antihypoxic and defense reactions. At the present time a number of various ways of sportspersons' special physical capability (SPC) based mainly on training and competitive loadings ramp up. They are effective enough to reach the main goal, but none of them provides sportspersons' health safety. Moreover, with the increase in volume and intensity of the loadings, which in sport have almost reached their limits, the sport traumatism and morbidity rate grow progressively. Proceeding from this, there was an evident necessity for the search of conceptually new ways for a simultaneous solution of these two the most complex and, in the opinion of many research workers, almost incompatible problems - the problem of achieving the highest levels of special physical working capacity, and the problem of sportspersons' health maintenance and improving -associated by us into one general problem of human motor activity efficiency enhancement. Therefore it is necessary to physiologically substantiated the basic methods and principles of special relaxation training, directed on increase of athletes training process efficiency at all stages of the athletic skills development. Under the efficiency of the motor activity, we understand achieve the highest levels of the special physical performance while maintaining sports longevity and health of athletes. In the conclusion we will note that it is necessary development of a brand new complex system of special physical and functional training, the use of which from the early child age will provide the all-round development and perfection of inhibitory-relaxation processes, one's own defence mechanisms and formation of the best rational types of long-term adaptation and individual development for an organism.
\end{abstract}

Keywords-extreme conditions; protection functional system; muscle relaxation rate; physical efficiency; central nervous system; relaxation.

\section{INTRODUCTION}

Currently, special attention is given to myorelaxation (muscle relaxation) as a non-traditional means of influence on the functional state of the human body. Myorelaxation is characterized by such features as: safety in exercising such influence, relative simplicity in the achievement of the necessary effect and low financial expenses. According to some authors, relaxation is also deemed as an alternative or additional means of the bodily functional state correction $[1,10,12]$. Relaxation methods have been used to correct a number of pathological conditions, treat a hypertensive disease, relieve acute and chronic pain, including those associated with sports activities [2, 3, 12].

\section{LiterATURE REVIEW}

The state of relaxation is also at the bottom of meditation techniques. There is a wide variety of the meditation and relaxation exercises applications; they are most often used in transcendental medicine $[5,6,10]$.

All the mentioned effects of relaxation methods are, without doubt, of great importance in sports activities as well. In this regard, a special emphasis needs to be made on such relaxation techniques as biofeedback, functional music, aromatherapy $[8,9,11,12]$ are recently developed based on the objective impact on the functional state of the athletes body.

Professional tendencies of the last years are connected with steady growth of loadings in practically all kinds of human professional activities. The consequence of this is often the disturbance in the work of regulatory mechanisms, that essentially decreases the level of physical capability and can result in various unfavorable vegetative shifts in health state [1, 2, 3], the problem of providing effective training of sportspersons in extreme conditions of life activity and creating functional preconditions for health saving being more and more topical. One of the ways to solve this problem is attracting modern effective and physiologically substantiated technologies with the 
physical, hypoxic and hyper-pyretic loadings; in the

simultaneous use of the functional state correction and complex diagnostics rational system. Such an approach allows widening the diapason of the body compensatory abilities against the maximal volume and intensity of professional and psycho-emotional loadings. The provision of optimal adaptation to muscular loadings can appear one of the conditions for the health level maintenance and professional mastery quality increase $[2,4,5,6]$.

Certainly, the given problem acquires a special meaning in modern conditions of the human professional activity. It finds its reflection in a series of works connected with the idea of loading criticism both in sport and other areas of professional activity $[6,7]$.

Together with traditional approaches a great experience of using a whole range of non-traditional means (midlands, baro-chamber, hypoxic and hyper-pyretic effects, special breathing exercises, methods of biological feedback, methods of active self-adjustment and relaxation, etc.) within the system of sport training is accumulated. Together with that it is necessary to note that among the non-traditional means of effect on the functional state of the human body a careful attention has lately been paid to myorelaxation methods, which such features as action safety, relative easiness of effect achieving and not high financial expenditures are typical of. Relaxation, on some authors' opinion, is considered as an alternative or compliment to the functional state correction $[8,9,10]$. That is why it is often presented as a means of prophylaxis, correction and emotional stresses elimination. There, as many note [10], it is the leading one in the series of methods allowing achieving necessary changes in the body's functional state.

In physiology an active process of muscular tone and psycho-emotional tension decrease $[9,11,12]$ are meant by relaxation. At relaxation there appears a trophotropic state, the level of anxiety, psychological and physiological response to stress effects decreases. Besides, relaxation is attended by a considerable reduction of afferent and efferent impulsation. As a consequence we can speak on the fact that the introduction of relaxation methods aimed at the prophylaxis, correction and negative psychoemotional states elimination into practice can promote adaptive capabilities of the body [2, 13, 14].

The relaxation methods found their application in the correction of a range of pathological states, hypertensive disease treatment, acute and chronic painful states taking down inclusive of sport activity $[10,15,16]$.

The state of relaxation lies in the foundation of meditative methods. Meditation and relaxation exercises have a wide diapason of application, most often they are used in transcendental medicine [4, 9, 11, 13].

The value of muscles relaxation function in human sport and labour activities is difficult to overestimate. In a series of works $[2,8,13,17,18]$ a healthy influence of special exercises enhancing the function of skeletal muscles relaxation on the central nervous system, visceral organs' and systems' activities, rational blood circulation types formation, motion coordination, tempo, stamina, technical skills, special physical working capacity and sport results growth were proved.

The investigations proving the leading role of inhibitory systems of the central nervous system and skeletal muscles' arbitrary relaxation rate (ARR) in the most important manifestations of life activity of the whole body: in the mechanisms of timed and long-tern adaptation to more mechanisms of heart adaptation and various blood circulation types formation; in the mechanisms of muscles blood supply and muscular activity energy supply; in the mechanisms of physical overwork stability improving, prevention of risks, traumas and diseases, and also in the body's mechanisms of defence from extreme conditions or factors and sportspersons rehabilitation [7, 10, 16, 19], are especially meaningful, in our opinion.

It should also be noted that all the most effective methods of psycho-regulation, self-adjustment and autotraining used in special psychological preparation of sportspersons and the latest health-improving technologies $[9,11,14]$ are based on relaxation.

\section{RESEARCH METHODOLOGY}

To study the mechanisms of regulation and coordination of arbitrary movements control the contractile and some relaxation characteristics of skeletal muscle, functional state of the central nervous system (CNS) and neuromuscular (NMS) systems we used the method of computer polymiografy, designed by $\mathrm{Y}$. Vysochin. It is used for training athletes of Russia and St.-Petersburg national teams. Marked indicates its high informativeness and reliability [2].

Polymyography is based on synchronous graphic recording of the bioelectric activities and strengths (in the form of the elektomyogram and the dynamogram, respectively) of both thighs quadriceps muscles during their maximally rapid and strong contraction and relaxation in the isometric mode. When deciphering polymyograms, we estimated the rates of the contraction and relaxation motor reactions; the rate of development and the strength of excitatory and inhibitory processes in the CNS; the inhibition-excitation balance in the CNS; the relative rate of voluntary muscle contraction, or the so-called explosive characteristics of muscles; the relative maximum voluntary muscle strength; the rate of voluntary muscle relaxation; and the general functional states of the muscles, CNS and NMS. In addition, we used the ratio between the relative maximum voluntary muscle strength and the rate of voluntary muscle relaxation to calculate the classification index of the long-term adaptation or individual development type.

\section{IV.RESULTS}

Series of experiments were attended by about 600 athletes of different skills. Summarizing the results of our longstanding research we can substantiate the main ways and principles of special relaxation training, aimed at higher effectiveness athletes training process at all stages of sport skills development. Under effectiveness of motional activity we understand achievement of the highest levels of special exercise performance (SEP) at condition of total preservation and improvement of athlete's health.

Summarizing the results of our longitudinal research we can substantiate the main ways and principles of special relaxation training, aimed at higher effectiveness of athletes training process at all stages of sport skills development. Under effectiveness of motional activity we understand achievement of the highest levels of SPC at condition of total preservation and improvement of athlete's health.

Nowadays there arc known different means of athlete's SPC improvement, based basically on increasing of 
achievement of the highest levels of exercise performance and preservation of health in extreme conditions at the same time. We also ascertained that heightened excitability of CNS is the main factors limiting the capacity of IRFPS $[3,11,20]$.

The relaxation type of individual development is the most profitable in all intents. For relaxation type persons the CNC exciting and inhibitory processes' balance, high rate of muscles' relaxation, excellent regulation and movement coordination, perfect reaction to moving objects, that guarantees the sport, everyday and street traumatism minimization, are specific. The most economical - eukinetic circulation type prevails in them, the cardiac performance high economical efficiency, the minimal level of energy consumption, a decreased concentration of energy exchange metabolites in blood, a high rate of reparative processes and resynthesis of energy resources, excellent physical performance and stamina prevail in them. They excel with an increased stress tolerance, twice or trice as seldom they are subject to overwork and diseases, as compared to the hypertrophic type persons. Relaxation type athletes, as contrasted with hypertrophic type ones, enjoy considerably greater sport longevity, stand physical and psychological stresses far easier, are subject to various overworks, traumas and diseases 8-10 times as seldom and achieve the highest sport results $[5,8,11]$.

With the increase VVR and the formation of long-term adaptation relaxation type the sport traumatism decreases progressively from $95-100 \%$ (at the VVR less than $4,01 / \mathrm{sec}$ ) to $5-0 \%$ (at the VVR more than 9,01/sec) and, therefore, their health improves the same progressively. Our multiyear investigations testified that even in the most traumatic kinds of sport, one can almost fully make away with injuries (except for the traumas emerging at gross violation of game rules by the rival) due to the correct organization of the work-out session aimed at the CNC nervous processes' balance normalization, muscles' VVR increase and long-term relaxation type formation.

In the next series of experiments 320 schoolchildren and qualified athletes (aged from 6 to 32) took part. As an adaptogenic factor a veloergometric exercise of maximal intensity was used.

At the age of 6-11 already a very high muscles' VVR was registered. Then it gradually decreased and by 14 years old became minimal, having deteriorated by $22,3 \%$. After 14 the muscles' VVR started gradually increasing again up to the age of 29 , and the early age (6-11) VVR level was achieved only by 20-25. The age-dependent dynamics of the IRFPS was analogous. Then it progressively decreased (by $12,6 \%$ ) achieving its minimal values by $13-15$ years old. After 14-15 years old the IRFPS capacity increased and by 23-25 years old took its peak level, and by 29 years old decreased a little. The same character of these parameters' dynamics was observed in women as well, only their decrease at the age of $13-15$ was less vividly expressed [4, 7, 20, 21, 22].

\section{CONCLUSION}

The above-mentioned facts, in our opinion, are quite important for understanding of the miorelaxation role in increase of SPC in all kinds of sport activities, because in each of them there are very high requirements in velocity, velocity tolerance or coordination, or different combination of these qualities, which directly depend on muscles VVR. of adaptation develops at athletes with high VVR of muscles and highly active IRFPS, and it provides 
PRESS

It should also be said here about the ARR highly authentic correlation relationships with all the principal components of motion coordination and sport results in various sports. The data for a significant influence of the ARR on the contractile muscles' properties realization degree also deserve attention. The enumerated facts, from our point of view, are meaningful enough to understand that important role, which is played by myorelaxation in the special physical capacity growth in all kinds of sport activity and sportspersons' health maintenance.

We assume that the listed facts are significant enough to understand the importance of myorelaxation in the growth of special physical working capacity in all types of sports activities, since each of them imposes higher requirements either to speed, speed endurance, coordination, or for various combinations of these qualities which directly correlate with arbitrary relaxation rate.

It is also necessary to note the need for the new integrated training for a new integrated training system for physical and functional progress applicable from the early training stages to secure a multisided progress with persistent improvements in the bodily relaxation and inhibition skills and processes and natural protection mechanisms - to form the most efficient type of long-term relaxation-driven adaptability and secure individual progress.

In the conclusion we will note that it is necessary development of a brand new complex system of special physical and functional training, the use of which from the early child age will provide the all-round development and perfection (training) of inhibitory-relaxation processes, one's own defence mechanisms and formation of the best rational types of long-term adaptation and individual development for an organism.

\section{References}

[1] Balsevich V.K. Human ontokinesiology. M.: Theory and practice of physical culture. 2000, p. 275.

[2] Vysochin Yu.V. Physiological mechanisms of defence, stability and physical working capacity improving in extreme conditions of sport and professional activity: Thesis of Dr. Sc. (Medicine). L.: HMA named after S.M. Kirov. 1988, p. 550.

[3] Denisenko Yu.P Relaxational characteristics of skeletal muscles in increase of physical working capacity of football players. Sport Science Bulletin. 2007, 1, p. 27-30.

[4] Vysochin Yu.V., Lukoyanov V.V. Active myorelaxation and selfadjustment in sport: Monograph -SPb.: SAPC naed after P.F. Lesgaft. 1997, p. 85.

[5] Denisenko Yu.P. Myorelaxation in football players' training system: Synopsis of thesis of Dr. Sc. (Biology). M. 2007, p. 48.

[6] Platonov V.N. Adaptation in sport - Kiev: Zdorovye. 1988, p. 257.

[7] Vysochin Yu.V., Denisenko Yu.P. Modern ideas on physiological mechanisms of terminable adaptation of sportspersons' body to physical loadings effects. Theory and practice of physical culture. 2002, 7, pp. 2-6.

[8] Aivazyan T.A. Relaxation therapy using biological feedback in treatment of hypertension patients. Biomanagement. Theory and practice. Novosibirsk. 1988, pp. 133-141.

[9] Sentyabrev N.N. Body's directed relaxation at intensive human muscular activity - Volgograd: VSAPC, 2004, p. 142.

[10] Tkhorevsky V.I. Blood supply of skeletal muscles at static and dynamic work. Candidate's thesis. M. 1967, p. 24.

[11] Vysochin Yu.V., Denisenko Yu.P. Health as national property. Collective monograph. SPb.: SAPC naed after P.F. Lesgaft. 2010,p. 669.

[12] Huxley H.E. The mechanism of muscle contraction. Science. 1969, vol. 164, p. 1356-1366.

[13] Vysochin Yu.V., Denisenko Yu.P., Yatsenko L.G. The contemporary concepts of structural and functional organization of the neuromuscular system and of the contraction and relaxation mechanisms of skeletal muscles. Pedagogico-pshychological and medico-biological problems physical culture and sport. 2011, vol.
4(21). URL: http://kamgifk.ru/magazin/journal.htm.-

[14] Sudakov K.V. Main principles of functional systems' general theory. Functional systems of the body: Guidance / under the editorship of Sudakov K.V. M.: Medicine. 1987, pp. 26-49.

[15] Bayevsky R.M., Motylyanskaya R.Ye. Cardiac rhythm in sportspersons M.: P\&S. 1986, pp. 144.

[16] Vysochin Yu.V., Denisenko Yu.P., Yatsenko L.G. Physiology, medicine, pharmacology, high technologies, the theory, practice: the collection of articles of the Fourth international scientifically practical conference «The high technologies fundamental and applied researches in physiology, medicine, pharmacology». SPb. 2012, vol. 1, p.p. $188-190$.

[17] Steg G. Efferent muscle innervation and rigidity. Acta Physiol. Scand. 1964 , vol. 61, 225, pp. 52 .

[18] Vallbo A.B. Cutaneous mechanoreception. Sixth Internationa Congress of Electromyography in Stockholm, Sweeden. 1979, pp. 1419.

[19] Vysochin Yu.V. Myorelaxation in mechanisms of orthopedic injuries. Sport and Nation's health: Collection of scientific works, SPb. 2001, pp. 74-84.

[20] Kuznetsova Z.M., Ryabchuk V.A., Labeshchenkov O.V. Theoretical and practical aspects of engineering profile students airborne training individualization. The Russian Journal of Physical Education and Sport. 2018, vol. 13(2), pp. 159-164. DOI: 10.14526/02_2018_326.

[21] Alexsandr S. Kuznetsov. Russian Professor's meeting. Russian Journal of Physical Education and Sport. 2019, 14(1), pp. 17-22. DOI: 10.14526/2070-4798-2019-14-1-18-24

[22] Denisenko Yu.P., R.A. Gumerov, Morozov A.I., Mardanov A.Kh. SPECIAL PHYSICAL WORKING CAPACITY BUILDING BY RELAXATION PRACTICES. Theory and practice of physical culture. 2018,9 , pp. 69-72 